\title{
Injection of energetic protons during solar eruption on 1999 May 9: Effect of flare and coronal mass ejection
}

\author{
J. Torsti ${ }^{1}$, L. Kocharov ${ }^{1}$, D. E. Innes ${ }^{2}$, J. Laivola ${ }^{1}$, and T. Sahla ${ }^{1}$ \\ 1 Space Research Laboratory, Department of Physics, University of Turku, Turku 20014, Finland \\ 2 Max-Planck-Institut für Aeronomie, 37191 Katlenburg-Lindau, Germany
}

Received 7 August 2000 / Accepted 20 October 2000

\begin{abstract}
A solar energetic particle (SEP) event was observed on 1999 May 9 by the Energetic and Relativistic Nuclei and Electron instrument (ERNE) onboard the Solar and Heliospheric Observatory (SOHO) spacecraft in association with a coronal mass ejection (CME) and X-ray flare at the western limb. Near flare onset the active region coronal loop structure was seen to erupt and simultaneous blue and red shift velocities of the hot plasma were recorded by the SUMER (Solar Ultraviolet Measurements of Emitted Radiation) instrument onboard SOHO. We observe for the first time three periods of the SEP injection in a single event: (i) the first, extremely-hard spectrum injection triggered by the passage of the flare initiated coronal (shock) wave; (ii) a moderately-hard spectrum phase starting about half a hour later, proceeding and ceasing concurrently with metric continuum radio burst; (iii) a prolonged soft spectrum injection dominating in the late phase of the event, after about $1.5 \mathrm{~h}$ from the first proton production. The CME bow shock acceleration provides a straightforward explanation of the final spectral redressing, whereas the first acceleration seems triggered by the flare. These observations lead us to conclude that the 1999 May 9 SEP event was caused by a combination of coronal and interplanetary acceleration processes contributing with varying importance at different stages of the solar eruption associated with both flare and CME. Comparison with other events suggests that it is a common property of mixed SEP events.
\end{abstract}

Key words. acceleration of particles - sun: flares - sun: corona - sun: particle emission

\section{Introduction}

During solar eruptions, flares and CMEs, particles from the ambient thermal plasma $(<10 \mathrm{keV})$ can be accelerated up to energy $>1 \mathrm{MeV}$ and escape into the interplanetary medium where they produce a SEP event at $1 \mathrm{AU}$. Acceleration of protons up to the SEP energy range may take place at the flare site and at the interplanetary shock wave driven by the CME (Reames 1999). Recent studies indicate that SEPs may be produced also on a global coronal scale between the impulsive flare and the interplanetary shock (Kocharov et al. 1999; Laitinen et al. 2000; Klein \& Trottet 2000). Several different acceleration regions may contribute to the SEP production during a single event as the eruption evolves from the low corona to the interplanetary medium (Cane et al. 1988; Cliver 1996). However, the relative contribution of different accelerators is still under discussion (e.g. Kahler et al. 2000).

It was observed in the angle-distant events that SEPs may have rapid access to remote $\left(>90^{\circ}\right)$ solar longitudes in association with chromospheric Moreton waves (Cliver et al. 1995) or coronal (EIT) waves

Send offprint requests to: L. Kocharov, e-mail: leon.kocharov@srl.utu.fi
(Torsti et al. 1999). Also in the case of well-connected SEP events with a double-peak structure, coronal shocks may contribute to the early component, whereas the last production is caused by the interplanetary shock wave driven by the CME (Torsti et al. 1996; Laitinen et al. 2000).

The solar eruption that occurred around 18 UT on 1999 May 9 was associated with a soft X-ray flare and a CME, which were both well observed by $\mathrm{SOHO}$ and YOHKOH. We employ spectral observations of the flare plasma carried out by the SUMER instrument (Wilhelm et al. 1995), and also the LASCO CME observations (Brueckner et al. 1995) to obtain plasma flow parameters. The associated proton event was detected by the ERNE instrument (Torsti et al. 1995) after 18:30 UT. In this work we analyze intensity-time profiles of accelerated protons measured in the energy range $1.8-120 \mathrm{MeV}$. Our aim is to find out candidates for the solar processes dominating the energetic proton injection into interplanetary space at various stages of the eruption. The harnessing of SUMMER data has enabled a more precise timing of the explosion start than would be possible with low cadence EIT images (e.g. Klassen et al. 2000) employed in our previous study (Torsti et al. 1999). 


\section{Analysis of proton data}

The particle instrument ERNE has two telescopes composed of several solid-state Si detectors, and CsI and BGO scintillators. They measure the nuclear charge and kinetic energy of particles in the range from $\sim 2$ to 100-500 MeV/nucleon depending on the charge. The energy range for protons is from 1.6 to $130 \mathrm{MeV}$. The 1999 May 9 proton event was observable in the entire energy range of ERNE. We use 1-min observation time bins. The energy determination of protons with energy $>12 \mathrm{MeV}$ is based on a ground-computer analysis of the pulseheight data during the first $20 \mathrm{~min}$ from the onset of the event, which gives a precise estimate for the velocity. For all the $<12 \mathrm{MeV}$ protons, and $>12 \mathrm{MeV}$ protons after 18:50 UT, we use on-board intensity observations in fixed energy channels. The number of energy channels is 10/decennium.

Position sensitive high-energy ( $>12 \mathrm{MeV}$ ) detectors are capable of determining the direction of incidence of charged particles, so that the proton pitch-angle distribution can be deduced with a high precision (e.g. Torsti et al. 1997). Then injection and interplanetary transport parameters can be estimated, using a theoretical model of interplanetary transport. For the goals of our present study, interplanetary scattering had been modeled as a pitch angle diffusion in the conventional slab model with spectral index $q=1.5$ and constant radial mean free path, $\Lambda_{\mathrm{r}}=$ constant (e.g. Kallenrode 1993), implemented in the numerical transport code AAS by Kocharov et al. (1998). We compared this with estimates from the simple scatterfree consideration that could be justified for the May 9 event because of the relatively large proton mean free path, $\Lambda_{\mathrm{r}}=0.7 \mathrm{AU}$. Both methods, anisotropy-intensity fitting and scatter-free estimates, yielded comparable results for the SEP injection parameters.

Intensity-time profiles of protons are shown in Fig. 1a. There are no significant simultaneous changes in count rates of different energy channels, which might be caused by spatial (local) effects. Magnetic field data also give no indications for abrupt changes that might locally affect proton intensity-time profiles (WIND/MFI observations). For these reasons, we regard significant details in the observed intensity-time profiles to be of a temporal nature. The first particles in the $50-100 \mathrm{MeV}$ range were observed at $\approx 18: 30$ UT. The time-of-flight estimate suggests the solar release time of the first particles at the root of the Earth-connected interplanetary magnetic line at $t^{(1)} \approx 18: 00 \mathrm{UT}$. A direct fitting of proton anisotropy and intensity-time profiles with the interplanetary transport model has refined this estimate: $t_{\text {shift }}^{(1)}=18: 10 \mathrm{UT} \pm$ $2 \mathrm{~min}$ (here the solar time is shifted by $+8.3 \mathrm{~min}$ to make comparison with near-Earth electromagnetic observations straightforward). Note that the remaining intensity levels from the previous event of 1999 May 3 (power-law shape with spectral index $S=-5.6$ ) are considerable in the low energy range, but they have decayed below the galactic background level above $15 \mathrm{MeV}$ at the time of the May 9

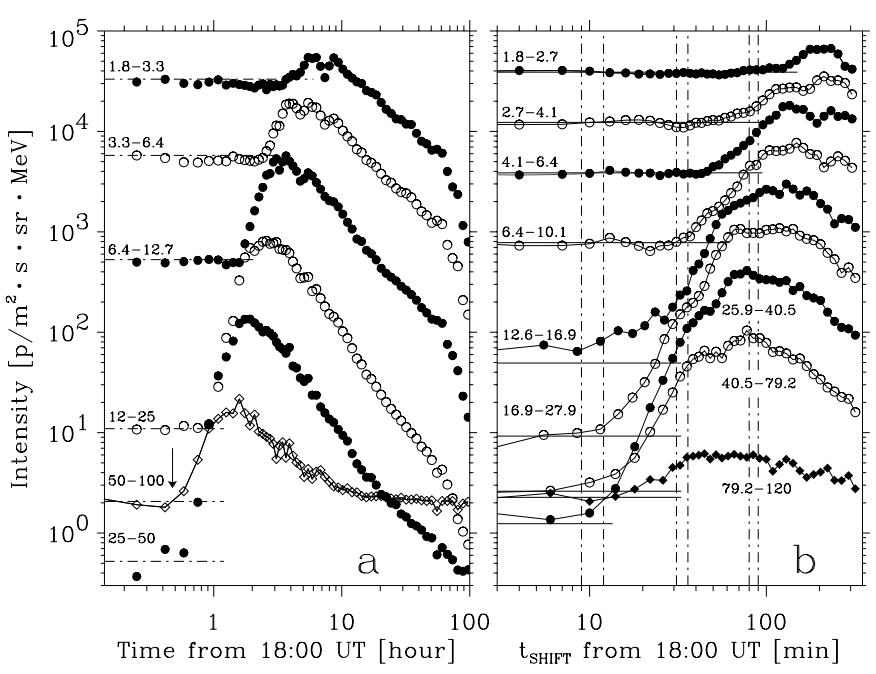

Fig. 1. a) Intensity-time profiles of protons during 100-hour period from 18:00 UT on 1999 May 9. Intensity background levels, averaged over 17-18 UT, are shown in each channel as horizontal lines. Channel limits in $\mathrm{MeV}$ are shown next to the curves. The arrow shows the latest crossing of the background level in the highest energy channel. b) Intensity profiles of protons as a function of the shifted time. Vertical line pairs show onsets of three periods of solar injection

eruption. For this reason, onset of the May 9 event in highenergy channels is dictated mainly by velocity dispersion, whereas below $10 \mathrm{MeV}$, the onset is additionally delayed due to the high background level from the previous event.

To account for the velocity dispersion and to make the comparison in different energy channels feasible, we follow the simple method previously employed by Krucker et al. (1999). We subtract from the observation time of particles in each energy range the travel time from Sun to Earth along the magnetic field line, and obtain a shifted time:

$t_{\text {shift }}=t_{\text {obs }}-L / V+8.3 \min$.

Here, $t_{\mathrm{obs}}$ is the time of particle observation, $L$ is the length of a particle flight trajectory from Sun to Earth, and $V$ is the particle velocity. The observed proton intensities are plotted as a function of the shifted time (Fig. 1b). We have adopted $L=1.3 \mathrm{AU}$. That value was found to bring the onset times in all five high-energy channels to the same moment of shifted time, $t_{\text {shift }}^{(1)}=18: 09-18: 12$ (see first vertical line pair in Fig. 1b).

In the high-energy channels 12.6-16.9, 16.9-27.9, 25.940.5, and 40.5-79.2 MeV, intensity-time profiles turn out to be downward concave around $t_{\text {shift }}=30-50 \mathrm{~min}$, but they start a new rise after that. We interpret this as a start of the second injection period between 18:31-18:36 shifted time (see second vertical line pair in Fig. 1b). The intensity rise in energy channels $25.9-40.5$ and $40.5-79.2 \mathrm{MeV}$ is somewhat delayed, because the first injection had a very hard spectrum, but the new one is much softer. To verify this interpretation, we have subtracted background intensities observed just before the event in the four high-energy channels, and then fitted the residual proton intensities 
at a certain $t_{\text {shift }}$ with a power law function $E^{S}$. The resultant proton spectral index as a function of shifted time is plotted in Fig. 2b. It is seen that the first injection had an extremely hard spectrum, $S \approx-1.5$. Then a fast softening of the injection spectrum starts around 18:36 shifted time, close to the second injection time estimated by eye from Fig. 1b. Note that the intensity rise at around 18:3118:36 is distinguishable also in three low-energy channels, from 2.7 MeV to $10.1 \mathrm{MeV}$ (Fig. 1b), but in these channels the rise starts from the enhanced level caused by the previous event. It is essential that there is a stationary value of spectral index during the second injection period, $S \approx-2.5$ (seen in Fig. 2 b at 55-85 min after $18 \mathrm{UT}$ ).

The third intensity rise is observed $80-90 \mathrm{~min}$ after 18 UT (see the third line pair in Fig. 1b). The enhancement is seen only in the low energy channels from $2.7 \mathrm{MeV}$ to $25.9 \mathrm{MeV}$. The event maximum intensities are reached 120-250 min after 18 UT. Again we ascribe the intensity change as a new injection phase starting between $\approx 19: 20$ 19:30 shifted time. It is seen from Fig. $2 \mathrm{~b}$ that the change brings further spectral softening which finally leads to a prolonged soft-spectrum production with spectral index $S \approx-3.5$.

Very similar results have been obtained with the direct fitting method. We have fitted anisotropy and intensitytime profiles in two energy channels, 16-20 MeV and 45$70 \mathrm{MeV}$. The injection profiles have been modeled as a sum of $N$ elementary injections, each exponentially rises and then exponentially decays, with adjusted characteristic rise/decay and maximum-injection times (similar to Laitinen et al. 2000). The profiles can be satisfactory fitted with the minimal number of the elementary components $N=3$. The deduced injection profiles are shown in Fig. 2c. They visualize three periods of injection with successively reducing values of the proton spectral index $S$. One can see only minor inconsistencies in timing between results shown in Figs. 2b and c. Those come from the value of $L$ in Eq. (1) which in fact slightly rises in the course of the event as more scattered particles arrive.

\section{Associated electromagnetic emissions}

On 1999 May 9, the SOHO/LASCO-C2 coronograph recorded a large CME centered in the northwest sector of the Sun. It was first seen in the 18:27 UT frame at about $2.5 R_{\odot}$ from the photosphere (plane-of-sky distance) at position angles around $295^{\circ}$. The CME height-time plot is shown in Fig. 2a. The first point in this figure corresponds to the 18:27 UT observation. The rest of the points in Fig. 2a are taken close to the CME nose at position angle $315^{\circ}$. The height-time plot is consistent with a uniform velocity of $540 \mathrm{~km} \mathrm{~s}^{-1}$ between 2.5 and $19 R_{\odot}$.

The CME was associated with an impulsive soft $\mathrm{X}$-ray flare in the active region NOAA 8537 situated near the west limb at latitude $\approx \mathrm{N} 20^{\circ}$ (position angle $\approx 290^{\circ}$ ). The hard X-ray pulse peaked at $\approx 17: 58 \mathrm{UT}$ and was about four minutes long (CGRO observations). Simultaneously

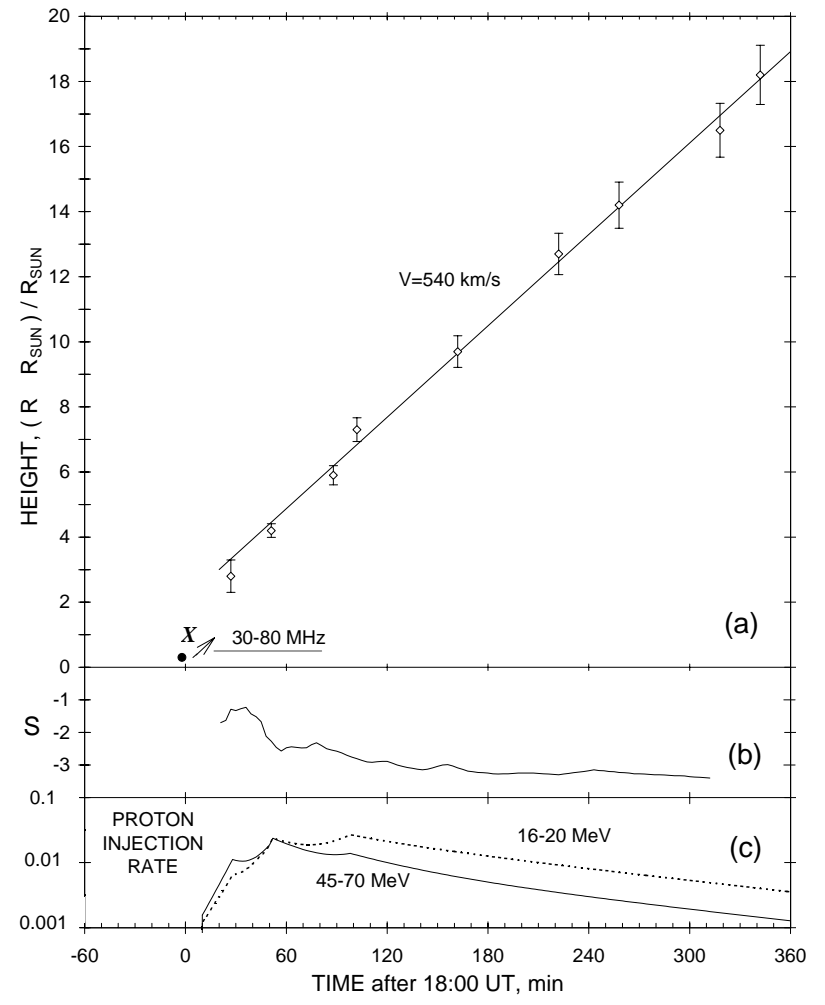

Fig. 2. a) The CME rise profile as observed by LASCO on 1999 May 9. For the X-ray flare $(X)$ we show position of the hard $\mathrm{X}$-ray peak (point) and motion of the soft X-ray ejecta (arrow). Radio continuum burst $(30-80 \mathrm{MHz})$ is indicated with a horizontal bar. b) Development of the proton spectral index as estimated from the intensity vs. shifted-time profiles. c) Proton injection profiles deduced by fitting anisotropy and intensity-time profiles in two energy channels (normalized to the same injection rate at the peak of the second injection)

the tall coronal loop $\left(\sim 0.15 R_{\odot}\right.$; well seen in the EIT $195 \AA$ images) erupted, and a fast bright optical emission front was seen against dark corona (Innes et al. 2000). Plasma acceleration over a wide range of temperatures is confirmed by the ultraviolet spectra taken by SUMER at the time of loop eruption. There are large Doppler-shifts at all positions along the spectrometer slit overlapping with the erupting loop and the optical emission front. The spectrum shown in the Fig. 3a contains a line from Fe XX representative of $10^{7} \mathrm{~K}$ plasma taken between 18:00:5618:05:56 UT. Both blue and red shifts are seen. The blue shift velocities are very high, up to $\approx 650 \mathrm{~km} \mathrm{~s}^{-1}$. At exactly the position of high blue and red shifts in the Fe XX emission line, the soft X-ray difference image of Yohkoh shows hot X-ray emission expanding northwards with a velocity $200 \mathrm{~km} \mathrm{~s}^{-1}$. This structure appears at the base of a hot corridor running from the top of the original loop system to, in this image, hot X-ray ejecta moving outwards into the corona (Fig. 3b). The ejecta velocity was $550 \mathrm{~km} \mathrm{~s}^{-1}$, which coincides, probably by chance, with the deduced velocity of the CME leading edge observed by LASCO at heights $>2.5 R_{\odot}$ (Fig. $2 \mathrm{a}$ ). 

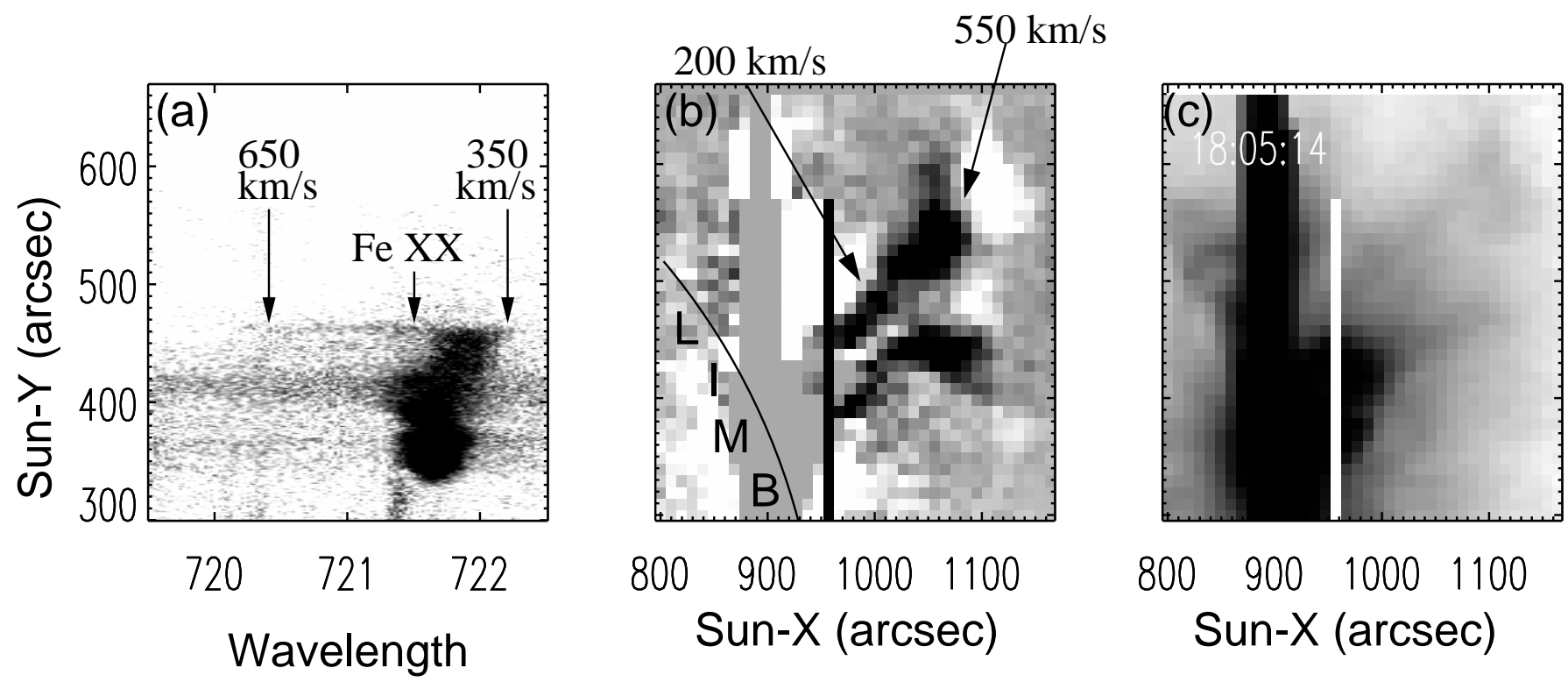

Fig. 3. a) SUMER spectrum of the Fe XX emission observed between 18:00:56 and 18:05:56 UT, showing line-of-sight plasma flows close to the flare onset time. The maximum blue and red Doppler velocities are indicated. The spectrum is measured at different coordinates Sun $-Y$ along the SUMER slit (see panel b). The wavelength is in $\AA$. b) The difference in soft Xray emission between 18:04:14 and 18:05:14, observed with SXT on Yohkoh. Black (white) signifies lower (higher) soft X-ray intensity in the later image. The position of the SUMER slit is marked as a vertical black line at Sun $-X=960$ arcsec. The plane-of-sky expansion speeds of two of the brightest soft X-ray emission sites are given: material is expanding outwards into the corona with a speed $550 \mathrm{~km} \mathrm{~s}^{-1}$ and northwards with a speed of $200 \mathrm{~km} \mathrm{~s}^{-1}$. c) Soft X-ray image taken at 18:05:14. The position of the SUMER slit is marked as a white line

A type III radio burst was observed at 18:03 UT (SGD 1999). No type II burst was identified. The most prominent feature is continuum radio emission enhancement in the $30-80 \mathrm{MHz}$ band recorded at Sagamore Hill Solar Radio Observatory starting 18:17 UT and ending 19:21 UT.

\section{Discussion}

As a reaction of the CME liftoff, a huge impulse must be imposed on the solar corona. If the impulse has been imposed during a sufficiently short time, the reaction force is also huge and a coronal wave is produced. Impulsive CMEs, often associated with flares and Moreton waves, move uniformly across the $2-30 R_{\odot}$ field of view of the LASCO coronographs (Sheeley et al. 1999). These CMEs that reach full speed in a short time should experience a strong acceleration below $\sim 2 R_{\odot}$, and the association with flares and Moreton waves seems natural. Generation of shocks and MHD turbulence as a reaction of CME and magnetic field line opening create all the necessary conditions for effective production of SEPs on a global coronal scale in association with the CME liftoff. Such a coronal acceleration is expected to operate during the first 1-2 hours of the eruption, preceding a prolonged period of CME bow shock acceleration in the interplanetary medium.

Cane et al. (1988) concluded that the highest intensities of the western events are due to the prompt solar particles above $20 \mathrm{MeV}$, but at below about $10 \mathrm{MeV}$ the CME shock-accelerated particles have higher intensities than the solar particles. That conclusion did possess a potentiality for developing a multistage paradigm of SEP acceleration. In contrast, a recent "black-and-white" paradigm decouples solar coronal acceleration and interplanetary CME acceleration (Kahler et al. 2000). Furthermore, these authors assume that posteruptive soft $\mathrm{X}$-ray arcades are the main candidate for coronal acceleration, antithetical to the CME bow shock acceleration in the interplanetary medium. It is surprising that we are listed among the authors who have suggested this, referring to Kocharov et al. (1994) and Torsti et al. $(1996,1998)$. The assumption that posteruptive arcades should produce significant SEP events even when the associated CMEs are too slow to drive shocks, was formulated and tested by Kahler et al. (2000). However this assumption has nothing to do with our ideas. Effective acceleration and escape of protons into the interplanetary medium requires strong enough MHD motions at open magnetic field lines. Both conditions should be met during the impulsive CME development in solar corona. The CME is an essential ingredient of the SEP production, irrespective of whether the CME bow shock is the only accelerator for high-energy protons or its coronal counterparts also accelerate protons. We have to recognize the facts that impulsive CME lift-off suggests corresponding coronal reaction, and the SEP production starts close to Sun (Shea \& Smart 1997) and crucially depends on 
the early evolution of the eruption below $\sim 2 R_{\odot}$ (Torsti et al. 1998). The 1999 May 9 SEP event provides a new evidence for coronal acceleration associated with flare pulse and CME liftoff.

The SUMER spectra of the flare show oppositely directed hot plasma flow in the FeXX line (Fig. 3). The blue Doppler-shifts correspond to plasma velocities $\approx 650 \mathrm{~km} \mathrm{~s}^{-1}$. The angular distance between the location of flare and the root of the nominal interplanetary magnetic field line connected to Earth was $32-38^{\circ}$ at the solar wind velocity $\approx 400 \mathrm{~km} \mathrm{~s}^{-1}$ (Wind/SWE observations). If we adopt the hard X-ray peak time, 17:58 UT, as the start of the flare eruption, we can expect that the plasma cloud itself, or more likely, an associated coronal shock wave, maintaining nearly the same velocity as the original plasma eruption, passed the foot area of the Earth connected field tube between $\approx 18: 09-18: 10$ UT. Our estimate for the onset of the ERNE observed proton injection, 18:10 UT \pm 2 min shifted time, fits well with the scenario that coronal wave injects first protons on arrival at the Earth-connected solar longitude. A similar scenario was suggested for disc flares associated with coronal Moreton (EIT) waves, where the first injected protons also had a very hard spectrum (Torsti et al. 1999).

We observe fast spectral evolution of energetic protons at the beginning of the May 9 event (Fig. 2b). The proton spectrum softening proceeds in two steps, separating three injection periods. The first injection is traced back to the flare eruption and was of the hardest spectrum, $S \approx-1.5$, while the final injection tends to $S \approx-3.5$. The movie of the LASCO coronagraph shows propagation of the leading edge of the CME from its first appearance at height $\approx 2.5 R_{\odot}(18: 27 \mathrm{UT})$ to the distance of $\approx 19 R_{\odot}$ at 24 UT. Comparing to the well-known result of Kahler (1994), that is interplanetary shocks are most effective in the injection of energetic protons at distances 4-10 $R_{\odot}$ from the Sun, we find that the peak of the third injection on 1999 May 9 corresponds to the $\mathrm{CME}$ distance $\approx 7 R_{\odot}$ (Fig. 2a) in agreement with the previously deduced range. Therefore we can conclude that the interplanetary CMEdriven shock acceleration is an obvious source of the final (third) period injection.

The second period injection was of spectral index $S \approx$ -2.5 . The injection ceased simultaneously with the radio continuum burst in metric band (Fig. 2), very similar to the previously studied western events of 1990 May 24 and 1996 July 9 (see Fig. 1 of Torsti et al. 1996, and Fig. 6 of Laitinen et al. 2000, respectively). This supports an idea that the second acceleration period is related to vigorous reconfiguration of coronal magnetic field during/after the CME liftoff. We speculate that an ensemble of shock waves may be involved.

Note that as the source of high-energy protons, the flare itself is not a candidate for any injection period because the ERNE observed protons were injected into the interplanetary medium $\approx 10 \mathrm{~min}$ after the flare impulsive phase. Also the everlasting proton domination over heavier nuclei does not support their production in the flare.
However, the flare pulse does trigger the coronal wave and by this expedient the first acceleration starts far from the flare site. Recently Reiner et al. (2000) described the distinction between coronal and interplanetary shock waves. Manoharam et al. (2000) argued for the different speeds of coronal and interplanetary shocks in the 25 June 1992 event. Also Thompson et al. (2000) re-analyzed observations of the 24 September 1997 coronal Moreton waves and CME and concluded that the timing and location of the coronal Moreton waves appear to indicate an origin with the flaring region, but there are also indications that the development of the CME may occur in concert with the development of the flare wave.

It is interesting to note that according to the standard classification, the 1999 May 9 event should be ranked among impulsive events because the associated X-ray flare was of a short duration (about $0.5 \mathrm{~h}$ at the $10 \%$ level). However the event was also CME associated and the $\mathrm{H} / \mathrm{He}$ ratio is very large, $\approx 1000$ at $20 \mathrm{MeV} /$ nucleon, while a representative value of $\mathrm{H} / \mathrm{He}$ in typical impulsive events is $\sim 10$ (Reames 1995). In addition, the ERNE observations do not show any enhancement in ${ }^{3} \mathrm{He}$. For these reasons, one should consider this event as a mixed event, according to the extended classification by Cliver (1996). Our present analysis of the 1999 May 9 event and recent results for four other events (Torsti et al. 1999; Kocharov et al. 1999; Laitinen et al. 2000) support the idea that mixed characteristics of such events come from a combination of a variety of coronal and interplanetary acceleration processes: (i) post-impulsive-phase acceleration processes triggered by the flare pulse and the fast CME-liftoff, operating on open magnetic field lines in extended coronal region; (ii) the long-lasting CME-driven shock acceleration in the interplanetary medium. We also speculate that the first acceleration(s) may provide a seed population for the second one.

Acknowledgements. We thank LASCO team for the CME movie available in SOHO archive. The ERNE team thanks Academy of Finland for the grant. The SUMER project is supported by DARA, CNES, NASA and the ESA PRODEX program. The SOHO is an international cooperation project between ESA and NASA.

\section{References}

Brueckner, G. E., Howard, R. A., Koomen, M. J., et al. 1995, Solar. Phys., 162, 357

Cane, H. V., Reames, D. V., \& von Rosenvinge, T. T. 1988, JGR, 93, 9555

Cliver, E. W. 1996, in High Energy Solar Physics, AIP Conf. Proc. 374, ed. R. Ramaty, N. Mandzhavidze, \& X.-M. Hua (New York: Woodbury), 45

Cliver, E. W., Kahler, S. W., Neidig, D. F., et al. 1995, Proc. 24th Internat. Cosmic Ray. Conf., 4, 257

Innes, D. E., Curdt, W., McKenzie, D. E., et al. 2000, in preparation

Kahler, S., 1994, ApJ, 428, 837

Kahler, S. W., McAllister, A. H., \& Cane, H. V. 2000, ApJ, 533,1063 
Kallenrode, M.-B. 1993, JGR, 98, No. A11, 19037

Klassen, A., Aurass, H., Mann, G., \& Thompson, B. J. 2000, A\&A, 141, 357

Klein, K.-L., \& Trottet, G. 2000, Space Sci. Rev., in press

Kocharov, L. G., Kovaltsov, G. A., Kocharov, G. E., et al. 1994, Solar. Phys., 150, 267

Kocharov, L., Vainio, R., Kovaltsov, G. A., et al. 1998, Solar. Phys., 182, 195

Kocharov, L., Torsti, J., Laitinen, T., et al. 1999, Solar. Phys., 190, 295

Krucker, S., Larson, D. E., Lin, R. P., \& Thompson, B. J. 1999, ApJ, 519, 864

Laitinen, T., Klein, K.-L., Kocharov, L., et al. 2000, A\&A, 360, 729

Manoharan, P. K., Kojima, M., Gopalswamy, N., et al. 2000, A\&A, 530, 1061

Reames, D. V. 1995, Rev. Geophys. Suppl., 33, 585

Reames, D. V. 1999, Space Sci. Rev., 90, 413

Reiner, M. J., Kaiser, M. L., Plunkett, S. P., et al. 2000, ApJ, 529, L53
Shea, M. A., \& Smart, D. F. 1997, Proc. 25th Internat. Cosmic Ray Conf., 1, 129

Sheeley Jr., N. R., Walters, J. H., Wang, Y.-M., et al. 1999, JGR, 104, No. A11, 24739

Solar-Geophysical Data 1999, No. 659-Part I, No. 663-Part II

Thompson, B. J., Reynolds, B., Aurass, H., et al. 2000, Solar Phys., 193, 161

Torsti, J., Valtonen, E., Lumme, M., et al. 1995, Solar. Phys., 162,505

Torsti, J., Kocharov, L. G., Vainio, R., et al. 1996, Solar. Phys., 166,135

Torsti, J., Laitinen, T., Vainio, R., et al. 1997, Solar. Phys., 175,771

Torsti, J., Anttila, A., Kocharov, L., et al. 1998, GRL, 25, No. 14,2525

Torsti, J., Kocharov, L. G., Teittinen, M., \& Thompson, B. J. 1999, ApJ, 510, 460

Wilhelm, K., Curdt, W., Marsch, E., et al. 1995, Solar. Phys., 162, 189 